\title{
Use of interreflection and shadow for surface contact
}

\author{
CINDEE MADISON \\ University of Minnesota, Minneapolis, Minnesota \\ WILLIAM THOMPSON \\ University of Utah, Salt Lake City, Utah \\ DANIEL KERSTEN \\ University of Minnesota, Minneapolis, Minnesota \\ and \\ PETER SHIRLEY and BRIAN SMITS \\ University of Utah, Salt Lake City, Utah
}

\begin{abstract}
The interaction of light with surfaces results in a number of lighting effects that may serve as valuable visual cues. Previous research on shadows has shown them to be effective in determining the three-dimensional (3-D) layout of a scene, but interreflectionshave been ignored as cues for spatial layout. Interreflections as well as shadows may help to disambiguate the 3-D layout of objects by providing information about an object's contact with a surface. We generated computer images of a box on an extended textured ground plane that was either in contact with the ground or was slightly above the ground. Images were rendered for four conditions: (1) no shadow + no interreflection, (2) shadow only, (3) interreflection only, and (4) shadow + interreflection. A photometrically incorrect condition was also included. The participants rated the degree of contact for each image on a scale, which was used to generate receiver operating characteristic (ROC) curves and a measure of sensitivity. In the images with no shadows or interreflections, the participants performed at chance levels. Interreflections, shadows, and a combination of interreflections and shadows all resulted in high sensitivity for judging object contact. More important, information from shadows and interreflections can be combined, resulting in near-perfect judgment of surface contact. Interreflections and shadows can be effectivecues for object contact.
\end{abstract}

In everyday experience, one interacts with objects and must determine their relative positions. Determining the spatial relationships between objects in a scene is important for path planning, object avoidance, reaching, and grasping. There is a considerable body of knowledge regarding both quantitative and qualitative perception of relative depth between objects (Cutting \& Vishton, 1995; Sedgwick, 1986). Quantitative judgments, such as estimation and discrimination, are based on a representation of distance as a continuous variable. Relative judgments of depth can also be qualitative and based on a categorical representation of object relations. An object can be inferred to be in front of or behind another, without an explicit estimation of metric distance. For example, when one object occludes another, the resultant $\mathrm{T}$ junction is a monocular cue for a qualitative depth relationship. The focus of this paper is on another useful but less studied

This research was supported by NSF Grant SBR 9631682, NIH Grant EY 11507, and NSF Grant GER 9454163. Address correspondence to C. Madison, University of Minnesota, 75 East River Rd., Minneapolis, MN 55455 (e-mail: cindee@cyclops.psych.umn.edu). qualitative judgment-deciding whether or not two surfaces are in contact. Contact is useful for one's deciding if a region is the actual object or a part of the object, or if the object is detachable or graspable. Contact information can also be used to estimate absolute or relative distances both to and between objects. For example, by determining the contact between an object and a homogeneously textured ground plane, observers can quite accurately judge the distance to the object (Sinai, Ooi, \& $\mathrm{He}, 1998)$. So, what are the cues indicating contact, and are there any that may be particularly important for contact judgments, as distinct from other judgments of relative spatial relationships?

Different pictorial cues can be used for determining contact, primarily by generating reliable depth judgments to different surfaces. Reliable depth judgments rely on image measurements that are well correlated with surface features. Image variation associated with illumination change typically confounds the detection of surface features. Because illumination effects such as shadows, interreflections, and specularities are highly variable and are not tied unambiguously to object location, they have historically been considered as something to be discounted in 
studies of shape and depth perception in both human and computer vision. However, the interaction of light with objects results in a number of illumination effects that might be useful cues for surface attributes and relations. For example, shadows have been found to be useful for the determination of spatial layout. In children, shadows have been found to be effective in influencing depth and height judgments of an object on a plane (Yonas, Goldsmith, \& Halstrom, 1978). The displacement between a cast shadow and the casting object in an image grows with the relative depth between the two surfaces (Kersten, Knill, Mamassian, \& Bulthoff, 1996; Kersten, Mamassian, \& Knill, 1997). This displacement measure is very effective for correctly evaluating the three-dimensional (3-D) spatial layout of a scene.

Interreflection is a subtle lighting effect that may also provide information for surface relations. Interreflections result from light bouncing between multiple surfaces (see Figure 1). They are responsible for illuminating areas that are not directly illuminated by the light source. Interreflections also add to the color and luminance reflected by areas receiving both direct and indirect illumination. In trying to create 3 -D photorealistic computer images, accounting for interreflections has proven to be very important and has lead to radiosity and ray-tracing rendering techniques in computer graphics (Foley, van Dam, Feiner, \& Hughes, 1996).

Interreflections may be important in determining surface contact, partially because of their close relationship to shadows. Though they are the result of light transport, as opposed to light occlusion, Langer (1999) recently provided a useful insight into the relation between the two. Consider the intersection of two surfaces. Decreasing the angle between the surfaces also decreases the likelihood for a randomly placed light source to strike either surface; hence, the more likely it is for there to be a shadow. However, as the angle between the two surfaces decreases, any light source that does enter will result in more bounces between the surfaces and, hence, the more likely it is for there to be interreflection. This close relation to shadows, which are effective in relating spatial layout, suggests that interreflections may also be a source of information for determining spatial layout.

Interreflections seem visually to be rather subtle. Although shadows can also have subtle effects, they can be quite sharp and salient, especially on a sunny day. Might the greater subtlety of interreflections reduce their effectiveness as a contact cue? Gilchrist and Jacobsen (1984) have shown interreflections to be potentially useful. They constructed a world of one reflectance in which observers were able to distinguish a matte white room from a matte black room, even when they were both illuminated so that they had the same average luminance values. The difference was the luminance profile of the room. The matte white room had a greater number of ambient bounces (interreflections) than did the matte black room, resulting in a flatter profile, whereas the matte black room had a more variable profile. Another study showed that the visual system is able to discount the effects of interreflection in determining surface color, given 3-D knowledge of the surface arrangement (Bloj, Kersten, \& Hurlbert, 1999). Bloj et al. created a colored Mach Card, one side red, the other side white. In the concave condition, observers were able to discount the pink color's bleeding through to the white side, which was due to interreflection, thereby seeing the side as primarily white with a tinge of pink. In the convex condition, the pinkish color was not discounted, and the same surface (previously seen as whitish) was perceived as magenta colored, a change in material property. In computer vision, interreflection has also been shown to be useful in algorithms that identify edge discontinuities (Forsyth \& Zisserman, 1992; Nayar, Ikeuchi, \& Kanade, 1991).

Although we have experimental evidence that the visual system is sensitive to and can make use of some of the subtle effects of interreflections, are these effects also useful for judging contact? If so, what is the image information for determining contact? In order to answer these questions, it is important to dissociate the contributions of cast shadows from those of interreflections. We measured the ability of observers to reliably judge the contact between two surfaces under conditions for which

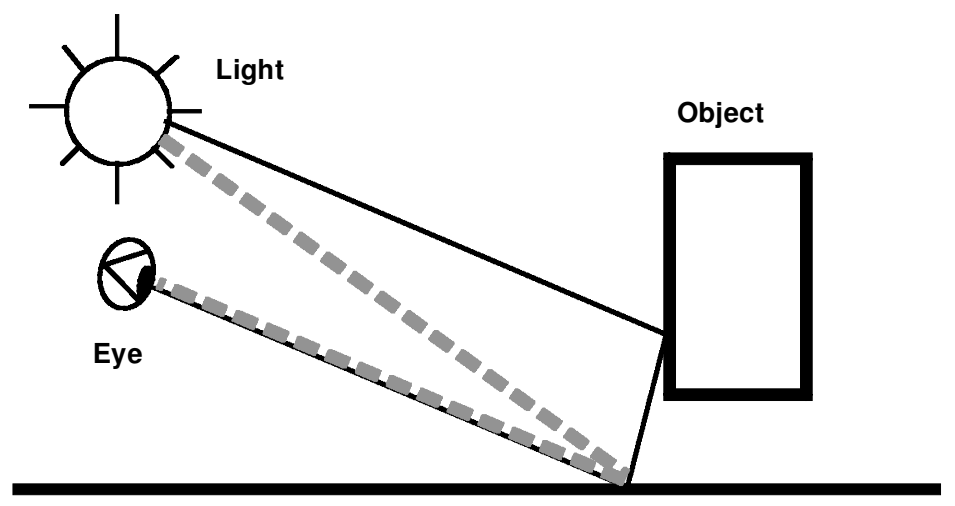

Figure 1. Interreflection. The dashed lines show direct reflection from a surface. The solid lines show the addition of light bouncing from a nearby object. 
we could control the contributions of both cast shadows and interreflections. Further, we also tested whether a simple geometrical constraint typical of certain types of contact might be important for contact judgments.

\section{METHOD}

Computer graphics were used to simulate images of a rectangular box that was either in contact with or was slightly above the floor (Figure 2). The floor was a continuous ground plane with a checkerboard texture. A checkerboard geometry provides strong cues to surface slant, whereas changes in reflectance create $\mathrm{X}$ junctions that provide additional information for determining illumination edges (Adelson, 1993; Knill, Mamassian, \& Kersten, 1997). Shadows and interreflections were generated by simulating the physics of light transport using a standard Monte Carlo renderer (Kajiya, 1986; Thompson, Shirley, Smits, Kersten, \& Madison, 1998). Observer viewpoint was fixed. The scene was illuminated by either one or two light sources, diffusely illuminating panels with flat intensity curves. Computed images were quantized to an image with 256 levels per channel, where zero intensity was mapped to the lowest image level, the largest RGB value was mapped to largest image level, and the remaining values were mapped linearly between these extremes.

We varied three basic conditions: light source (one or two), shadow (present or absent), and interreflection (present or absent). Figure 3 shows the 16 basic conditions in gray-level format.

In order to test whether a simple geometric constraint (discussed below) could be used to determine contact, we added a condition in which the image rendering was not based on a realistic model of the physics of light. This "faked" condition had one light source, a white shadow, and a photometric ally incorrect (red instead of green) interreflection (see Figure 4).

In each of the above conditions, the scene was rendered twice, once with the box slightly above the floor and once with the box touching the floor.
Twenty-two participants viewed each image 10 times in random order. The participants made judgments in which they ranked their confidence regarding the contact between the floor and the box. The degree of contact choices were: definitely touching, maybe touching, unsure, maybe above, and definitely above. The images were displayed at a resolution of $1,024 \times 768$ on an Applevision 1710AV display at a distance of $64 \mathrm{~cm}$. The monitor had a gamma of 1.8 , and the images were gamma corrected in order to use the standard Macintosh look-up table. The images had a mean luminance of $120 \mathrm{~cd} / \mathrm{m}^{2}$, with the shadow areas averaging $15 \mathrm{~cd} / \mathrm{m}^{2}$, and the highly illuminated areas averaging $200 \mathrm{~cd} / \mathrm{m}^{2}$. All the participants had normal or corrected-to-normal vision, gave their informed consent, and, for their participation, received extra credit in a research experience program connected to a first year psychology class.

\section{Data Analysis}

The participants' responses were analyzed independently. Because each image can be considered to have a "correct answer" (either touching or above), as determined by the geometry of the 3-D model, receiver operating characteristic (ROC) curves could be generated for each individual, for each condition. The ROC curve is a graph of hits versus false alarms for various criterion levels. The ROC can be generated by determining the correct number of responses at each criterion level (definitely touching, maybe touching, unsure, maybe above, and definitely above) and plotting hits (object is touching and the participant says it is touching) against false alarms (object is not touching and the participant says it is touching; Green \& Swets, 1974). The area under the ROC curve provides a measure of sensitivity and ranges from 0.5 (chance) to 1 (perfect). Our summary measure of contact was $z$ score of the area under the ROC curve. ${ }^{1}$

\section{Results}

Figure 5 shows the calculated sensitivity for the oneand two-light conditions. Standard error bars were cal-

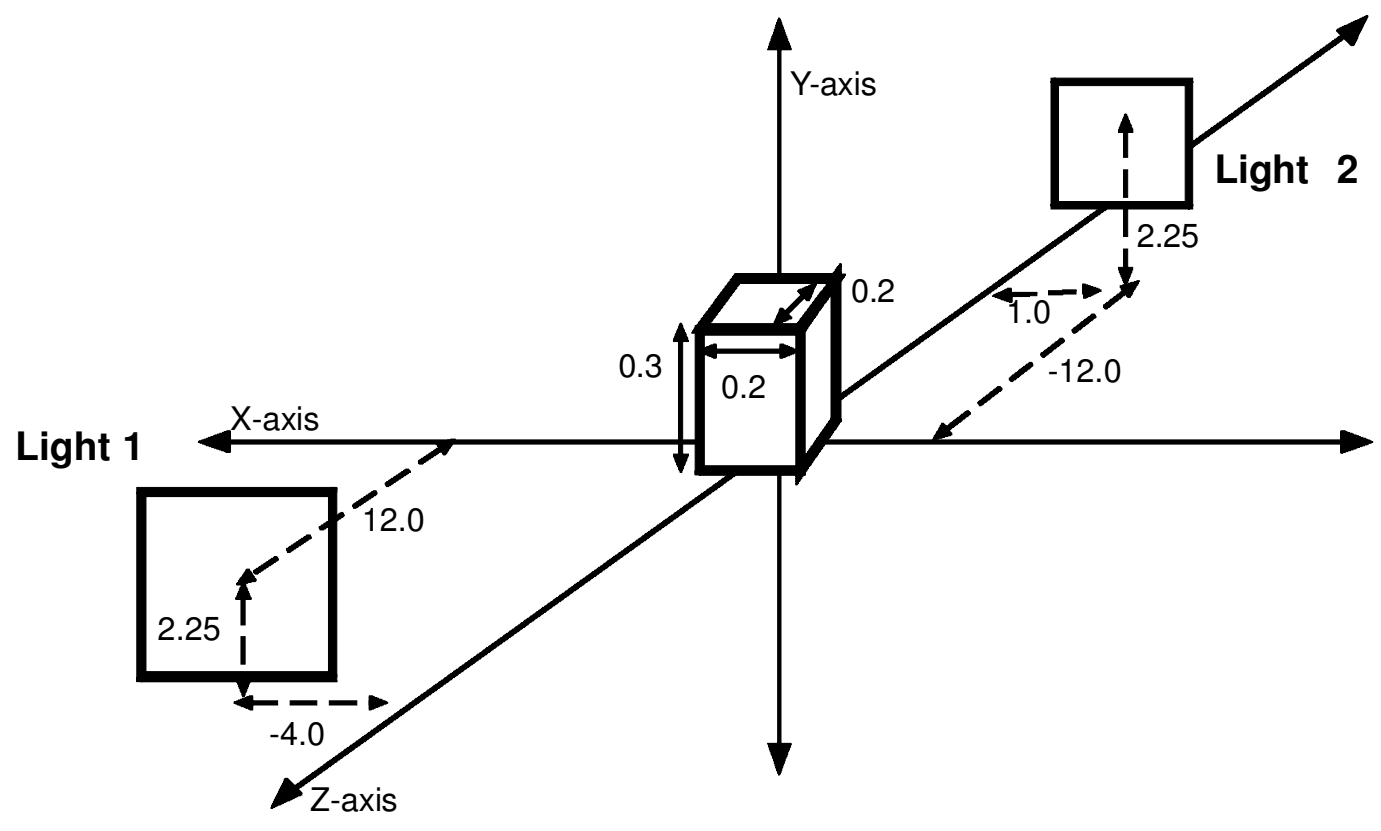

Figure 2. Geometry of the scene. The rectangular box is centered at the origin. Light 1 is centered at $(-4.0,2.25$, 12.0). Light 2 is centered at $(1.0,2.25,-12.0)$. Both lights are $1.5 \times 1.5$ units. In the touching condition, the rectangle lies on the floor plane $(y=0)$. In the above condition, it is 0.010 units above the floor plane. All measurements are in absolute units. 


\section{One-Light Source}

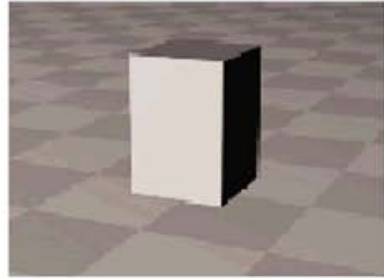

No Shadow

No Interreflection

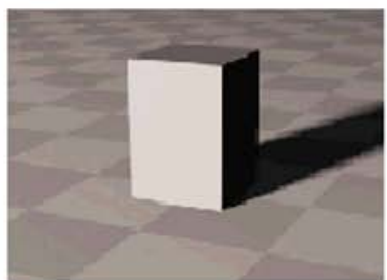

Shadow

No Interreflection

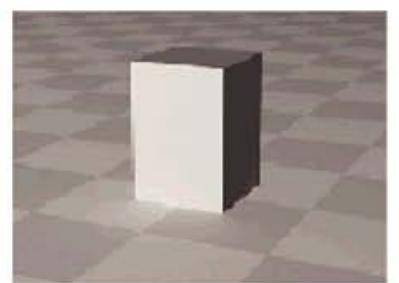

No Shadow Interreflection

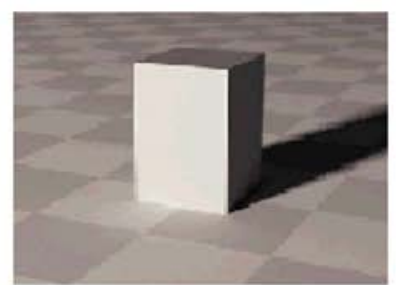

Shadow

Interreflection

Touching

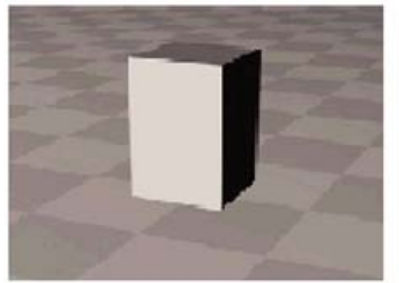

No Shadow

No Interreflection

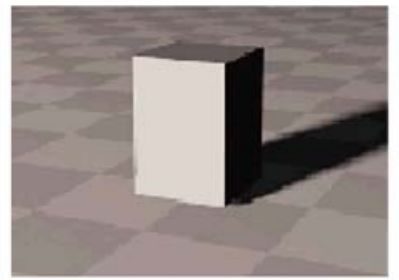

Shadow

No Interreflection

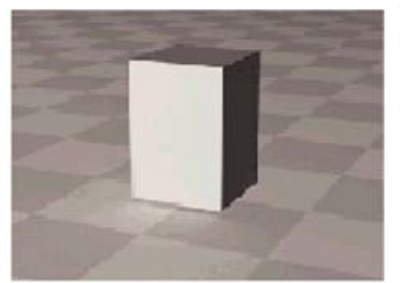

No Shadow

Interreflection

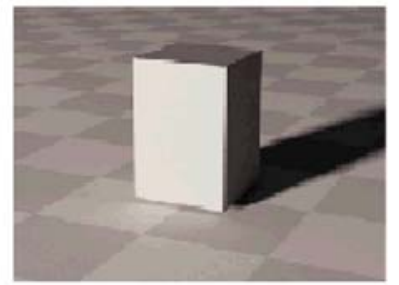

Shadow

Interreflection

Above

\section{Two-Light Source}

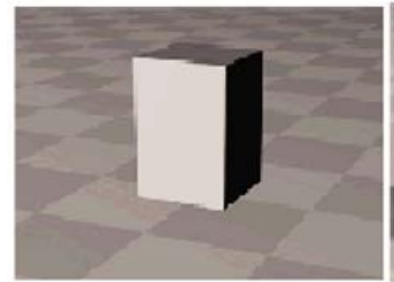

No Shadow

No Interreflection

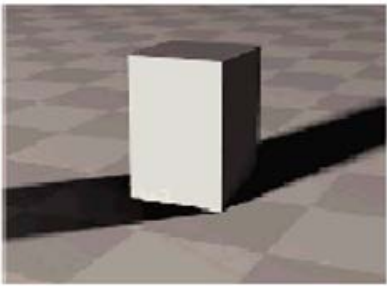

Shadow

No Interreflection

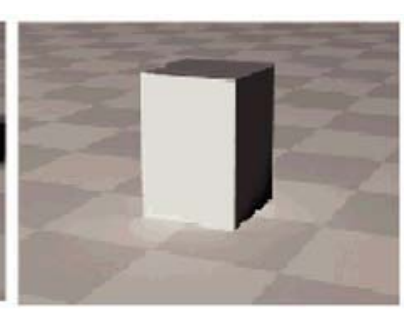

No Shadow

Interreflection

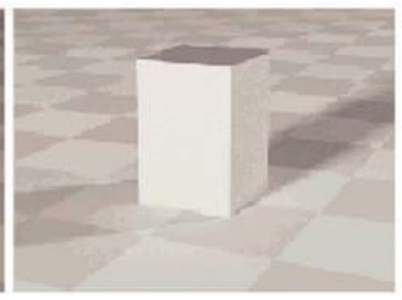

Shadow

Interreflection

Touching

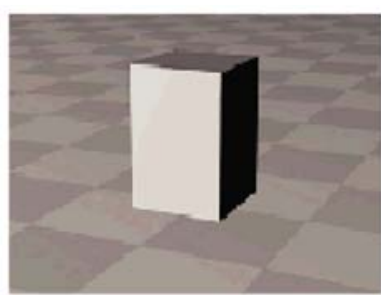

No Shadow

No Interreflection

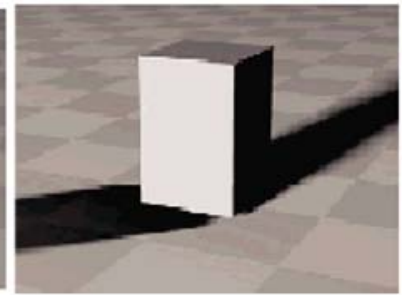

Shadow

No Interreflection

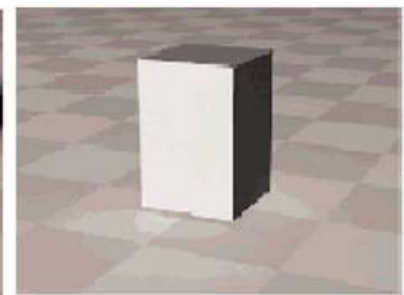

No Shadow

Interreflection

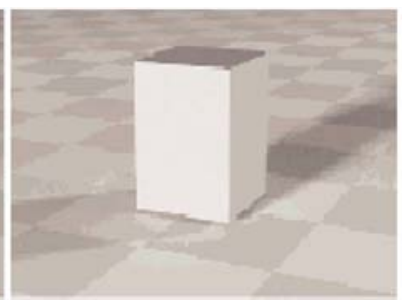

Shadow

Interreflection

Figure 3. Gray-level versions of images used in the experiments. (The original images are available at http://vision.psych.umn.edu/ www/people/cindee/glueimages.html.) 


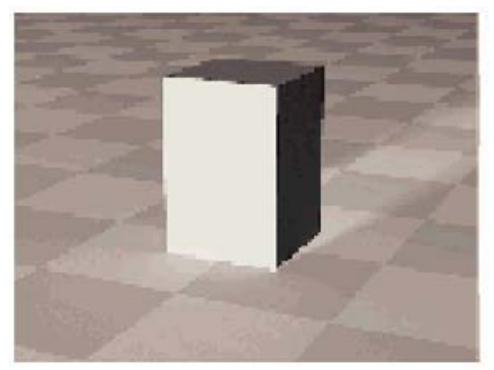

White Shadow

Red Interreflection Touching

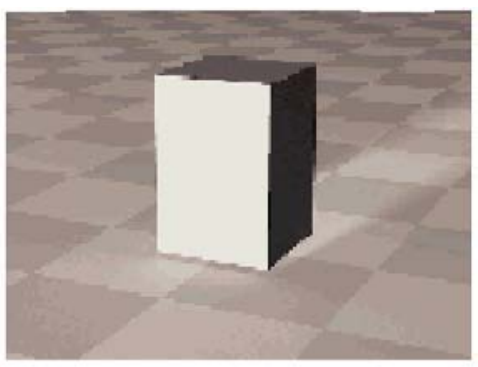

White Shadow

Red Interreflection

Above

Figure 4. Gray-level versions of "faked" images. These images had the wrong color interreflection (red instead of green) and the wrong polarity shadow (white instead of black). (The original images are available at http://vision.psych.umn.edu/www/people/ cindee/glueimages.html.)

culated by bootstrapping the original data 1,000 times, calculating the mean sensitivity $(z$ score of the area under the ROC curve) for each replication, and using the standard deviation of the replications (Efron \& Tibshirani, 1998). In the one-light condition, the participants were significantly better at discriminating contact using shadow information as compared with using interreflection information. However, in the two-light condition, performance in the shadow and interreflection condition was not significantly different. The participants did worse with the two-light-source shadows than with the onelight-source shadows; however, they did better with the two-light-source interreflections than with the singlelight-source interrreflections. In both the one-light- and two-light-source conditions, the combination of shadow and interreflection resulted in the highest sensitivity to contact. This sensitivity was significantly greater in the shadow + interreflection condition than in either condition alone. A natural question to ask is whether this improvement in sensitivity is due to probability summation or information summation.

Probability Summation. Probability summation is described by the summation of two detectors' output of the independently analyzed signals and is computed using the following equations:

$$
\text { Hits: } 1-\left(1-\mathrm{P}_{\text {hitS }}\right)\left(1-\mathrm{P}_{\text {hitt }}\right) \text {, }
$$

where $\mathrm{P}_{\text {hits }}$ is the probability of hit for the shadow condition, and $\mathrm{P}_{\text {hitI }}$ is the probability of hit for the interreflection condition;

$$
\text { False alarms: } 1-\left(1-\mathrm{P}_{\mathrm{faS}}\right)\left(1-\mathrm{P}_{\mathrm{faI}}\right) \text {, }
$$

where $\mathrm{P}_{\mathrm{faS}}$ is the probability of false alarm for the shadow condition, and $\mathrm{P}_{\text {faI }}$ is the probability of false alarm for the interreflection condition.

Once the probability summation of hits and false alarms is calculated, it can be used to generate an ROC curve, which is used to calculate the predicted sensitivity
( $z$ score of the area) to a combination of shadow and interreflection. The estimate of sensitivity resulting from probability summation is plotted in Figure 5.

Information summation. Information summation combines information from two independent sources more efficiently than does probability summation. Estimated performance on the shadow + interreflection condition is calculated using the following equation:

$$
d_{\mathrm{sum}}^{\prime}=\sqrt{\left(d_{1}^{\prime}\right)^{2}+\left(d_{2}^{\prime}\right)^{2}}
$$

where $d_{1}^{\prime}$ is the sensitivity measure for one condition (i.e., shadow), and $d_{2}^{\prime}$ is the sensitivity measure for a second condition (i.e., interreflection).

The predicted sensitivity due to information summation $\left(d_{\text {sum }}^{\prime}\right)$ is plotted in Figure 5.

The sample data fall right between the predicted sensitivity values of probability summation and information summation.

Because of the ceiling on probability summation and the large number of "expert" participants in this experiment, who showed perfect sensitivity in the task with just shadows or just interreflections, participants were split between expert and nonexpert groups. In the one-lightsource condition there were 5 shadow experts, 2 interreflection experts, and 9 shadow + interreflection experts. In the two-light-source condition there was 1 shadow expert, 5 interreflection experts, and 11 shadow + interreflection experts. The data for each group were reanalyzed to see whether the experts might have been combining the information from the stimuli in a different manner than the nonexperts. Resampling of the raw rating data with a bootstrap technique (Efron \& Tibshirani, 1998) provided statistical support for the conclusion that the participants showing perfect sensitivity in the task with just shadows or just interreflections by chance, would be extremely rare $(p<.005)$. An important point to note is that in both the one-light and two-light conditions, the 


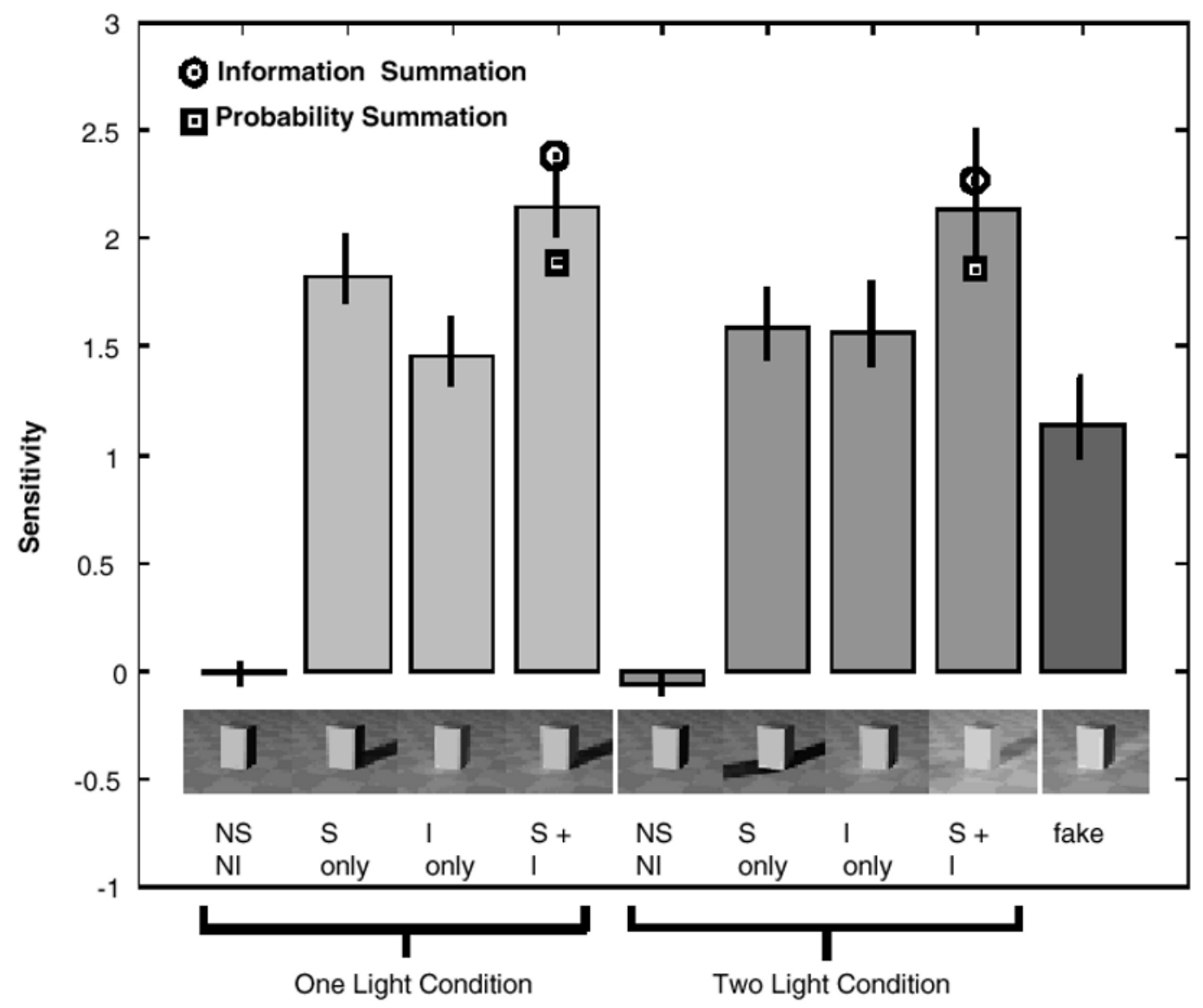

Image conditions

Figure 5. Sensitivity measures for each condition. The light bars are for the one-light condition. The medium bars are for the two-light condition. The darkest bars represent the "faked" condition (white shadows and photometrically incorrect interreflections). Sensitivity is measured in $z$ score of the receiver operating characteristic area under the curve (note 0.0 is chance). Error bars were calculated using bootstrap analysis. Predicted performance in the shadow + interreflection was based on probability summation (circle) and on information summation (square) and is plotted with the actual shadow + interreflection data. NS NI = no shadow + no interreflection; $S$ only $=$ shadow only; I only $=$ interreflection only; $S+I=$ shadow + interreflection.

combination of shadows and interreflections resulted in a greater number of experts than did the shadow or interreflection conditions alone. However, analysis suggests that the experts did not combine the information from the stimuli in a different manner than the nonexperts.

Faked images. Analysis of the faked-image condition shows that sensitivity for contact with white shadows and photometrically incorrect interreflections was less than with either the photometrically correct shadow-only or interreflection-only conditions. However, performance was still well above chance (see Figure 5). Although the participants were given contradictory photometric information, what information might have been available for their making the contact decision?

Image analysis. The images were analyzed in order to investigate possible geometrical cues that may be useful for a contact decision. An analysis of the isophotes, or lines of continuous luminance, shows a potential geometrical cue (see Figure 6).

In the touching condition, the isophotes of the interreflection all have a coincidental alignment at the corner of the box. This was also true for the edge of the shadow. However, in the condition where the box was slightly above the floor, there was no coincidental alignment.

\section{Discussion}

It is important to note that in the no shadows and no interreflections conditions, performance for determining object contact was at the chance level. The images for this case differed from each other only in the height of the box in the image plane. Without shadows or interreflections to provide useful cues for contact, the participants were unable to discriminate the touching boxes from the boxes above. This also suggests that in this par- 

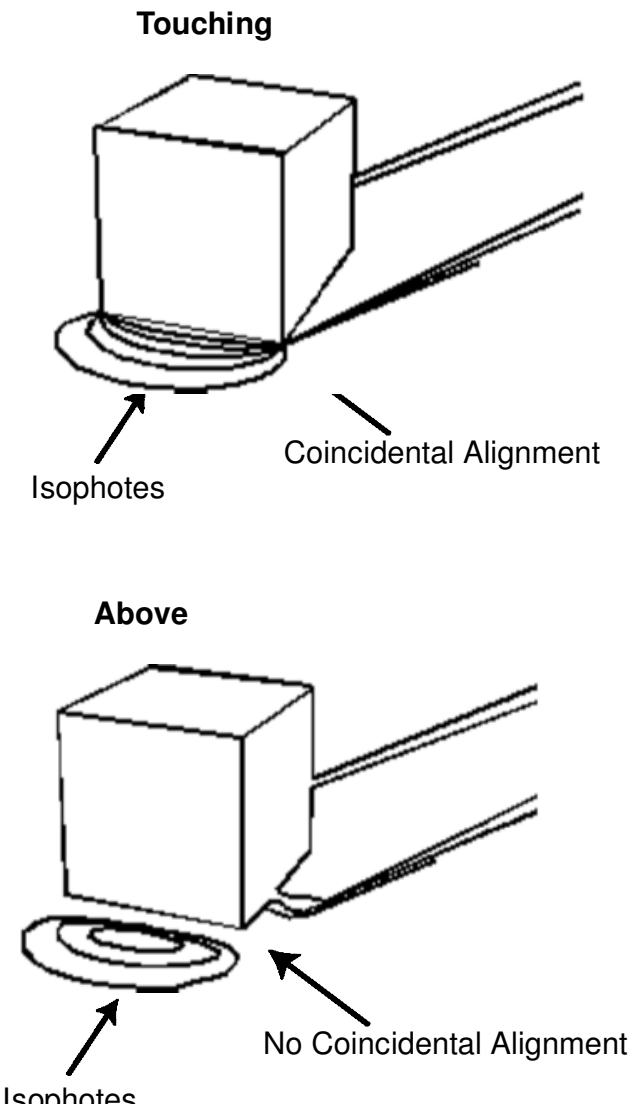

Isophotes

Figure 6. Image analysis in one-light source condition. Isophotes are the lines of continuous luminance and include interreflection isophotes, shadow isophotes, and shading isophotes.

ticular task, the participants did not have any systematic biases resulting from the effect of other cues (e.g., coplanarity with the floor plane). The addition of shadows or interreflections resulted in higher sensitivity to object contact. The decrease in sensitivity for discrimination with two-light-source shadows might have been due to the coincidental alignment of the shadows, which could have resulted in the participants' interpreting the shadows as pigment changes or (e.g., a rug) and not to an illumination effect. Improvement in the two-light-source interreflection condition might have been due to more geometrical cues to contact, as was addressed in the Image Analysis section of this paper. The combination of shadows with interreflections resulted in the highest sensitivity, which was significantly greater than that for the images that had only shadows or only interreflections. Though analysis of the data does not clearly point to either probability summation or information summation, it is clear that cue combination was occurring. The combined effect of shadows and interreflections resulted in much higher sensitivity to contact, suggesting the impor- tance of shadows and interreflections in making subjective contact decisions.

The faked condition, which combined shadow and interreflection, resulted in a relatively high sensitivity to contact. Although the illumination effects were not physically correct, the participants could still make accurate responses; however, sensitivity was much worse for this case than for the photometrically correct shadow-only and interreflection-only conditions. Image analysis provided one potentially useful cue in the geometry of the isophotes. The alignment of the shadow and/or the interreflection at the corner of the box might have provided some information for the contact decision. However, the most accurate judgment required both photometrically correct shadow and interreflection, suggesting that agreement of all cue information led to the best judgment.

\section{CONCLUSION}

Shadows have generally been assumed to be cues for contact; the present results validate that assumption and also introduce interreflection as an equally important cue for object contact. Although interreflections appear to be more subtle than shadows, under the conditions of the present experiment, they conveyed equally strong information about object contact. However, the greatest sensitivity resulted from a combination of both shadows and interreflections. In combination, shadows and interreflections prove to be valuable cues for determining contact and therefore also for determining spatial layout. The present study provides support for the growing importance of considering illumination effects in investigating human visual perception of spatial layout.

\section{REFERENCES}

Adelson, E. H. (1993). Perceptual organization and the judgment of brightness. Science, 262, 2042-2044.

Bloj, M. G., Kersten, D., \& Hurlbert, A. C. (1999). Perception of three-dimensional shape influences colour perception via mutual illumination. Nature, 402, 877-879.

Cutting, J. E., \& Vishton, P. M. (1995). Perceiving layout and knowing distances: The integration, relative potency, and contextual use of different information about depth. In W. Epstein \& S. Rogers (Eds.), Perception of space and motion (pp. 69-117). San Diego: Academic Press.

Efron, B., \& Tibshirani, R. J. (1998). An introduction to the bootstrap. New York: Chapman \& Hall.

Foley, J., van Dam, A., Feiner, S., \& Hughes, J. (1996). Computer graphics: Principles and practice, 2 nd edition in C. Reading, MA: Addison-Wesley.

Forsyth, D., \& Zisserman, A. (1992). Reflections on shading. In L. B.Wolff, S. A. Shafer, \& G. E. Healey (Eds.), Physics based vision: Principles and practice: Shape recovery. Boston: Jones \& Bartlett.

Gilchrist, A., \& JACOBSEn, A. (1984). Perception of lightness and illumination in a world of one reflectance. Perception, 13, 5-19.

Green, D. M., \& Swets, J. A. (1974). Signal detection theory and psychophysics. Huntington, NY: Krieger.

KAJIYA, J. (1986). The rendering equation. SIGGRAPH 1986 Proceedings, 20, 143-150. 
Kersten, D., Knill, D. C., Mamassian, P., \& Bulthoff, I. (1996). Illusory motion from shadow. Nature, 379, 31 .

Kersten, D., Mamassian, P., \& Knill, D. C. (1997). Moving cast shadows induce apparent motion in depth. Perception, 26, 171-192.

Knill, D. C., Mamassian, P., \& Kersten, D. (1997). The geometry of shadows. Journal of the Optical Society of America A, 14, 3216-3232.

LANGER, M. (1999). When do shadows become interreflections? International Journal of Computer Vision, 34, 1-12.

Nayar, S. K., Ikeuchi, K., \& Kanade, T. (1991). Shape from interreflections. International Journal of Computer Vision, 6, 173-195.

SEDGWICK, H. A. (1986). Space perception. In K. R. Boff, L. Kaufman, \& J. P. Thomas (Eds.), Handbook of perception and human performance (pp. 21-1 to 21-57). New York: Wiley.

SINAI, M. J., OOI, T. L., \& HE, Z J. (1998). Terrain influences the accurate judgement of distance. Nature, 395, 497-500.

Thompson, W. B., Shirley, P., Smits, B., Kersten, D. J., \& Madi-
Son, C. (1998). Visual glue (Tech. Rep. UUCS-98-007). University of Utah.

Yonas, A., Goldsmith, L.T., \& Halstrom, J. L. (1978). Development of sensitivity to information provided by cast shadows in pictures. Perception, 7, 333-341.

\section{NOTE}

1. The $z$ score of the area is proportional to $d^{\prime}$, a standard measure of sensitivity. Specifically, the area under the ROC curve equals the predicted proportion correct in a two-alternative forced-choice experiment, assuming equal-variance Gaussian distributions on the underlying decision variable.

(Manuscript received February 23, 2000; revision accepted for publication May 17, 2000.) 